\title{
Geschiebehaushalt in kleinen Hochgebirgsbächen der Nordtiroler Zentralalpen
}

\author{
Johannes Kammerlander · Stefan Achleitner · Johannes Schöber · Bernhard Hofer
}

Online publiziert: 6. März 2017

(c) Der/die Autor(en) 2017. Dieser Artikel ist eine Open-Access-Publikation.

\begin{abstract}
Zusammenfassung Kleine Hochgebirgsbäche sind bedeutende Sedimentlieferanten für die Talflüsse und beeinflussen deren Geschiebehaushalt und dadurch auch deren morphologischen und ökologischen Zustand. Allerdings ist wenig zu den Geschiebemengen von Hochgebirgsbächen bekannt. In der gegenständlichen Untersuchung werden Messdaten von 20 Hochgebirgsbächen der Nordtiroler Zentralalpen mit einem Beobachtungszeitraum von bis zu 49 Jahren präsentiert. Die Auswertung dieser Datenreihen zielt auf die Abschätzung von Jahresgeschiebefrachten

resgeschiebefrachten geringer als die jährliche Schwankung der Abflussverhältnisse. So wird in trockenen Jahren verhältnismäßig mehr Geschiebe transportiert (höhere Transporteffizienz) als in feuchten. Die Größenordnung der Jahresgeschiebefracht scheint demnach vor allem durch die jährlich begrenzte Verfügbarkeit von feinem Geschiebe reguliert zu werden und weniger durch den Jahreswasserabfluss. Ein alleiniger Zusammenhang zwischen Abfluss und Geschiebetransport ist in den untersuchten Hochgebirgsbächen demnach nicht gegeben.
\end{abstract} ab. Die Ergebnisse zeigen einen linearen Zusammenhang zwischen der mittleren Jahresgeschiebefracht und der Einzugsgebietsgröße und eine exponentielle Abhängigkeit zur Vergletscherung. Der Einfluss der Vergletscherung begründet sich dabei maßgeblich durch die hohe Verfügbarkeit von feinem $\mathrm{Ge}$ schiebe im Gletschervorfeld.

Neben der räumlichen weisen die Jahresgeschiebefrachten auch eine starke zeitliche Variabilität auf. Die Verteilungen sind durchwegs rechtsschief, was einem häufigen Vorkommen von Jahren mit relativ geringen Frachten und einem seltenen Vorkommen von ganz großen Frachten entspricht. Allerdings ist die Variabilität der Jah-

\section{J. Kammerlander $(\bowtie)$}

Institut für Naturgefahren,

Bundesforschungszentrum für

Wald, Rennweg 1, 6020 Innsbruck,

Österreich

j.kammerlander@gmx.at

DI J. Kammerlander .

DI Dr. S. Achleitner

Arbeitsbereich Wasserbau,

Universität Innsbruck,

Technikerstraße 13, 6020 Innsbruck, Österreich

Mag. Dr. J. Schöber · DI Dr. B. Hofer TIWAG,

Eduard-Wallnöfer-Platz 2, 6020 Innsbruck, Österreich
Variability of annual bed load volumes in small mountain creeks located in the Central Alps of Tyrol (Austria)

Abstract In an alpine environment, small mountain creeks represent the upper part of the fluvial network. Bed load fluxes originating from these headwater or tributary streams are an important sediment source for their receiving gravel bed streams and hence, they influence the sediment budget of the entire alpine river network. However, little is known about the bed load budget of such small mountain creeks.

The operational records of a hydropower operator are used to determine annual bed load volumes of twenty mountain catchments with a time span of up to 49 years. Correlation analyses of the annual bed load volumes with site-specific parameters are presented in the first part of the paper. Within-site variation of annual bed load volumes is log-normally distributed for most of the sites and their means are positively correlated with the catchment size and the relative glacier area.

Since the bed load budget of steep mountain streams is mainly controlled by water run-off, stream morphology and sediment availability, an approach is presented which enables to separate these driven factors in the second part.
Based on that, the between-site and within-site variability of transport efficiency is determined. Results suggest that sediment availability regulates the within-site variability of annual bed load volumes, at least during hydrological ordinary years. In this respect, transport efficiency is on average higher in dry years than in wet ones. Additionally, the impact of an exceptional flood event on bed load transport could be quantified at one site. The event caused an increase of bed load transport efficiency of about one order of magnitude which successively decreased to its former level within three years.

\section{Einleitung}

1.1 Geschiebetransport in Hochgebirgsbächen

Ein Gutteil der Sedimentproduktion eines alpinen Gewässers passiert in den Gebirgsregionen und es sind die zahlreichen kleinen Hochgebirgsbäche, welche die Talflüsse mit Geschiebe versorgen. Die Bedeutung der Geschiebedurchgängigkeit ist hinlänglich bekannt und sie stellt im Wasserbau eine wichtige Zielsetzung dar (Habersack et al. 2013). Der Geschiebehaushalt eines alpinen Gewässers ist entscheidend für dessen flussbaulichen und ökologischen Zustand.

Allerdings ist der Geschiebehaushalt in den Hochgebirgsbächen noch wenig erforscht. Dies trifft vor allem für den Geschiebetransport während hydrologischer Normaljahre zu, also ohne Auftreten von großen Hochwasserereignissen. Die Messung des Geschiebetransports in Gebirgsbächen gewinnt zwar zunehmend an Bedeutung, allerdings existieren noch sehr wenige Langzeitbeobachtungen. Die gängigen Methoden sind mobile Geschiebesammler (direkte Messung; Bunte et al. 2004) oder Geophone/Hydrophone (indirekte Messung; Rickenmann et al. 2014). Aber auch in den Entsanderanlagen von Wasserfassungen können die aufgetre- 
tenen Geschiebemengen quantifiziert werden (Tschada 1975; Hofer 1987).

Aus theoretischer Sicht ist der Geschiebetransport das Resultat einer überschüssigen Abflussenergie, mit welcher das Sohlmaterial in Bewegung versetzt und talwärts transportiert wird. Der Zusammenhang zwischen Strömungskraft und Geschiebetransport ist seit Mitte des letzten Jahrhunderts Gegenstand zahlreicher Untersuchungen und dem Ingenieur stehen ebenso zahlreiche Formelwerke für die Berechnung zur Verfügung. Die Mehrzahl dieser Untersuchungen beschränkte sich jedoch auf Laborversuche mit flachen Gerinnen und/oder Einheitskorn. Die daraus abgeleiteten Formelwerke eignen sich demnach bevorzugt für flache Talflüsse. Die Anwendung dieser Formeln für steile Gebirgsbäche erfordert die zusätzliche Berücksichtigung der komplexen Morphologie und der stark ungleichmäßigen Strömung (Nitsche et al. 2011).

Aktuelle Forschungen zeigen allerdings, dass sich die rechnerisch ermittelten Kubaturen dennoch häufig sehr stark von tatsächlich beobachteten unterscheiden (Klar 2016), vor allem in Bezug auf längere Zeitspannen mit langen Mittelwasserperioden. Das hat zum einen mit der großen Unsicherheit in der Erhebung der notwendigen Parameter zu tun, zum anderen aber auch mit der nach wie vor inadäquaten Abbildung der Prozesse. Die Abschätzung der Geschiebeeinträge aus kleinen Hochgebirgsbächen ist jedoch für Modellierungen von Talflüssen von großer Wichtigkeit, da sie eine primäre Geschiebequelle darstellen.

\subsection{Kornverteilung der Jahresgeschiebefracht in Hochgebirgsbächen}

In den 1970er-Jahren wurden von der TIWAG (und in Zusammenarbeit mit der Universität Innsbruck) umfangreiche Untersuchungen $\mathrm{zu}$ den Kornverteilungen von Hochgebirgsbächen durchgeführt (Sommer unveröffentlicht; Sommer und Lauffer 1982). Eine wesentliche Erkenntnis dieser Arbeiten war der erhebliche Unterschied zwischen der Kornverteilung des über einen gewissen Zeitraum transportierten Geschiebes und jener der Bachsohle.

Die Kornverteilung der Bachsohle von Hochgebirgsbächen ist in der Regel sehr grob und das Mittelkorn wie auch andere charakteristische Korngrößen stehen häufig in einem positiven $\mathrm{Zu}$ sammenhang mit dem lokalen Bachgefälle (Kammerlander 2017; Schneider et al. 2015a). Die Grobkomponenten der Bachsohle stellen maßgebende Rauheitselemente dar und beeinflussen somit den Fließwiderstand im Gerinne.

Die Kornverteilung des transportierten Geschiebes erweist sich hingegen als deutlich feiner. In den früheren Untersuchungen wird es auch als laufendes Geschiebe bezeichnet, was den Transport dieses Materials über die Deckschicht der Sohle versinnbildlicht (Sommer und Lauffer 1982). Zwar nimmt das Maximalkorn des transportierten Geschiebes in der Regel mit dem Abfluss $\mathrm{zu}$, allerdings ist dieses auch bei alljährlichen Hochwasserereignissen deutlich kleiner als jenes der Sohle bzw. mengenmäßig unterrepräsentiert.

\subsection{Wechselwirkung zwischen Geschiebeverfügbarkeit und Transporteffizienz}

Ein Ungleichgewicht zwischen der Kornverteilung des, beispielsweise in einem Jahr, angefallenen Geschiebes und des Sohlmaterials ist langfristig nur möglich, wenn die Geschiebequelle eine andere ist als die unmittelbare Bachsohle. Dies ist auch ein wesentliches Unterscheidungsmerkmal zu den Talflüssen (Kiesbettflüsse). In letzteren ist das Sohlsediment die primäre Quelle für den Geschiebetransport. Folglich sind auch die Kornverteilungen des transportierten Geschiebes und jene des Sohlmaterials ähnlich (Parker und Klingeman 1982).

In Hochgebirgsbächen stellt die Verfügbarkeit von relativ feinem, bei alljährlichen Abflussverhältnissen mobilisierbarem Sediment einen zusätzlich limitierenden Faktor dar. So ist die tatsächliche Geschiebeführung in steilen Gebirgsbächen häufig um ein Vielfaches geringer als das theoretische Transportvermögen (Sommer und Lauffer 1982). Man spricht in diesem Zusammenhang auch vom Zustand der latenten Erosion.

Dieser Untersuchung liegt die Hypothese zugrunde, dass die Geschiebeführung in einer bestimmten Gewässerstrecke nicht nur an den Abfluss, sondern auch an den Sedimenteintrag von bachaufwärts gebunden ist. Bei hoher Sedimentverfügbarkeit sollte demnach verhältnismäßig mehr Transport stattfinden als bei geringer (Kammerlander et al. 2017; Recking 2012). In anderen Worten: Die tatsächliche Transportrate nähert sich mit zunehmender Verfügbarkeit von feinem Geschiebe der theoretischen Transportkapazität an. In diesem Zusammenhang wird der Begriff der Transporteffizienz definiert. Es ist ein Maß für die Menge an Geschiebe die je Strömungseinheit tatsächlich transportiert wird.

Die Bedeutung der Geschiebeverfügbarkeit auf die Transporteffizienz lässt sich am Beispiel von alpinen Wasserfassungen eindrucksvoll aufzeigen. An solchen Bauwerken wird ein Gutteil des natürlichen Abflusses abgeleitet, während das transportierte Geschiebe zurückgehalten und periodisch in das Unterwasser abgegeben wird. Im Unterwasser steht demnach dieselbe Menge an Geschiebe einer deutlich reduzierten Wasserfracht gegenüber. Unter der Annahme eines starren Zusammenhangs zwischen Geschiebetransport und Strömungskraft, müsste es im Unterwasser solcher Bauwerke zu starken Sedimentablagerungen kommen. Die langjährige Erfahrung zeigt jedoch ein anderes Bild. Trotz der erheblichen Abflussreduktion wird das Geschiebe auch im Unterwasser weitertransportiert und starke Sohlanlandungen sind ausgeblieben (Tschada und Hofer 1990; Schönlaub und Hofer 2009). Von ähnlichen Beobachtungen in den USA berichtet auch Ryan (1997).

\section{Jahresgeschiebefrachten in den Hochgebirgsbächen der Zentralalpen}

Um nun Aussagen über den Geschiebehaushalt von Hochgebirgsbächen treffen zu können, sind langjährige Messdaten zu der Menge an (jährlichem) Transport erforderlich. $\mathrm{Zu}$ komplex sind die Vielzahl an beeinflussenden Faktoren um diese mittels theoretischer Berechnungen oder In-situ-Messungen der Transportrate abzuschätzen.

Umfangreiche Untersuchungen $\mathrm{zu}$ den Jahresgeschiebefrachten in Hochgebirgsbächen wurden bereits in den 1970er- und 1980er-Jahren durchgeführt (Tschada 1975; Sommer und Lauffer 1982; Schöberl 1983). Als Datengrundlage standen damals die Spülaufzeichnungen aus 14 Betriebsjahren von zehn alpinen Wasserfassungen der TIWAG zur Verfügung. Diese Wasserfassungen sind nach wie vor in Betrieb und wurden durch weitere Standorte ergänzt. Der Beobachtungszeitraum 


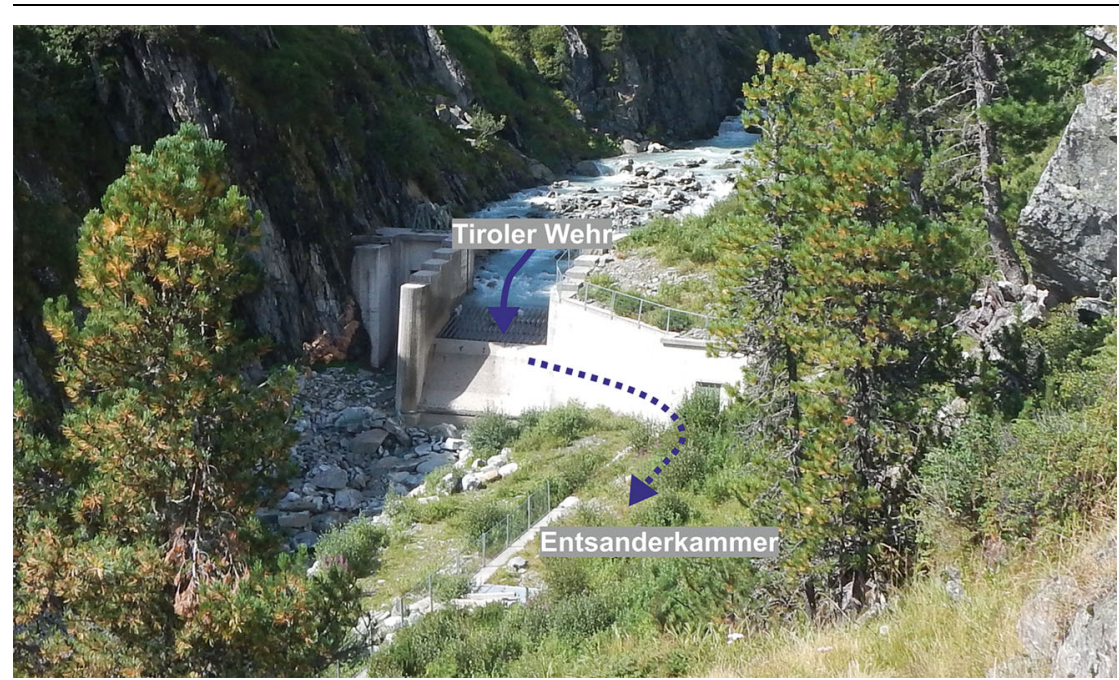

Abb. 1 Wasserfassung am Oberbergbach (Stubaital, Tirol)

Skizze einer Entsanderkammer

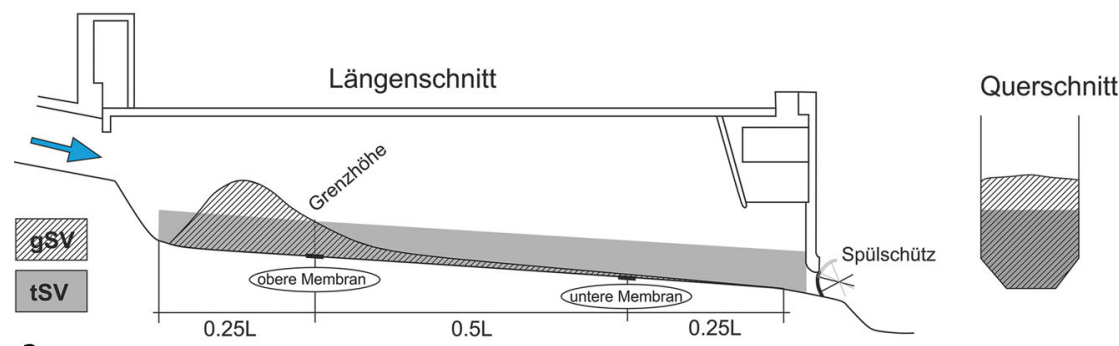

a

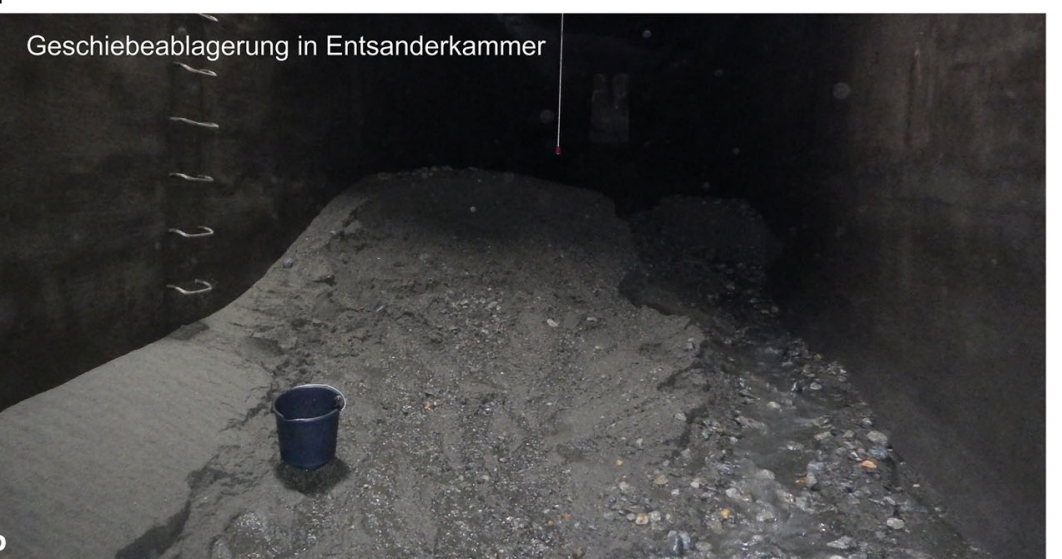

Abb. 2 a)Skizze einer Entsanderkammer, in der diemaximaleAblagerungshöheander oberen Membran erreicht ist; die graue Fläche entspricht dem theoretischem Volumen bei sohlparalleler Ablagerung und die schraffierte Fläche der wahren Ablagerungsform; b) Geschiebeablagerungskegel in der Entsanderkammer der Oberbergbachfassung

umfasst nun bis zu 49 Jahre, was eine Aktualisierung der Auswertung mehr als rechtfertigt.

\subsection{Alpine Wasserfassungen der TIWAG}

Im Rahmen des Kaunertalkraftwerks und der Kraftwerksgruppe Sellrain-Silz betreibt die TIWAG 20 Wasserfassun- werden. Dies erfolgt durch einen künstlichen Wasseraufstau, der geringe Fließgeschwindigkeiten und ebenso geringe Strömungskräfte zur Folge hat. Dadurch gelangen das eingezogene Geschiebe und ein Teil der Schwebstoffe zur Ablagerung, während das Wasser über ein Tauchwehr in den Ausleitungskanal geleitet wird.

Zur Vermeidung von zu großen Sedimentablagerungen sind regelmäßige Entleerungen notwendig, die in Form von Spülungen durchgeführt werden. Dazu wird die Entsanderkammer abgestaut und der eingezogene Abfluss wird durch die Entsanderkammer und den nachfolgenden Spülkanal in das Unterwasser geleitet. Das abgelagerte Sediment wird dabei mobilisiert und die Entsanderkammer gespült.

Während des Spülvorgangs kann somit kein Wasser in den Ausleitungskanal abgeleitet werden. Folglich führt ein zu häufiges Spülen zu einem erheblichen Verlust an Wassereinzug. Im Gegensatz dazu besteht bei seltenem Spülen die Gefahr, dass die Entsanderkammer zur Gänze mit Sediment verlandet und der Mechanismus des automatischen Freispülens zum Erliegen kommt. Zur Optimierung der Spülhäufigkeit entwickelte die TIWAG ein System, welches eine Spülung automatisch aktiviert, sobald die Sedimentanlandung ein kritisches Maß erreicht. Dazu sind am Kammerboden mehrere (in der Regel zwei) Sohlmembranen angeordnet, welche die Auflast der Sedimentablagerung messen. Sobald diese Auflast einen voreingestellten (kritischen) Wert erreicht, wird ein hydraulischer Mechanismus ausgelöst, der zu einer kurzzeitigen Öffnung des Spülschutzes führt (Schober 1961).

An den zahlreichen Wasserfassungen der TIWAG wird die Anzahl an Spülungen seit deren Inbetriebnahme dokumentiert. Diese Daten sind die Grundlage für die nachfolgend beschriebene Bestimmung der Jahresgeschiebefrachten von 20 Hochgebirgsbächen über einen Zeitraum von bis zu 49 Jahren.

\subsection{Methode zur Bestimmung der Jahresgeschiebefrachten} ren gefasst und in den nächstgelegenen Speicher geleitet wird (Abb. 1). Die Entnahmemenge ist dabei lediglich durch die Ausbauwassermenge beschränkt.

Dem Entnahmebauwerk (Tiroler Wehr) schließt jeweils eine Entsanderkammer an (Abb. 1), in welcher das Wasser und die Feststoffstoffe (mit Ausnahme der Schwebstoffe), getrennt
Zur Bestimmung der Jahresgeschiebefrachten ist zusätzlich zur jährlichen Anzahl an Spülungen auch die Kenntnis der mittleren Geschiebemenge je Spülung erforderlich. Die automatische Spüleinrichtung garantiert dabei, dass die Spülungen systematisch, bei Errei- 
Tab. 1 Gebietsspezifische Parameter zurBerechnung des Feststoffvolumensan Geschiebe zum Zeitpunkt einer automatischen Entsanderspülung (mGV) mit dem theoretischen Sedimentvolumen bei sohlparalleler Verlandung (tSV), dem Skalierungsfaktor (sf), der Porosität (e) und dem Geschiebeanteil (ge)
Tab. 2 TopografischeParameter derEinzugsgebiete (jeweils bachaufwärts der Wasserfassung) mit der Einzugsgebietsfläche (A), der Vergletscherung (VG), der mittleren Hangneigung $(\mathrm{mN})$, dem mittleren Bachgefälle des Gerinneabschnitts direkt oberhalb der Wasserfassung $(\mathrm{Ga})$, dem gesamten Hauptgerinne $(\mathrm{Hg})$, dem gesamten Gewässernetz (Gn) und von maßgebenden Flachstrecken im Hauptgerinne (Fs) sowie der Gewässerdichte (Gd)

chen eines Grenzwertes initiiert werden und nicht den zufälligen Schwankungen von Wartungsarbeiten ausgesetzt sind. In der Vergangenheit wurden da$\mathrm{zu}$ die Sedimentablagerungen in den Entsanderkammern unmittelbar vor

\begin{tabular}{|c|c|c|c|c|c|c|}
\hline \multirow[t]{2}{*}{ Bachname } & Zeitraum & tSV & sf & $\mathrm{e}$ & ge & mGV \\
\hline & & $\left(m^{3}\right)$ & $(-)$ & $(-)$ & $(-)$ & $\left(m^{3}\right)$ \\
\hline Schöntalbach & $1987-2013$ & 17.2 & $0.61^{b}$ & 0.3 & 0.65 & 4.7 \\
\hline Schelfalmbach & $1985-2013$ & 20.1 & $0.61^{b}$ & 0.3 & 0.65 & 5.5 \\
\hline Klammbach & $1985-2013$ & 33.3 & $0.61^{b}$ & 0.3 & 0.65 & 9.2 \\
\hline Unterer Liesenerbach & $1985-2013$ & 26.8 & $0.61^{b}$ & 0.3 & 0.65 & 7.4 \\
\hline Oberer Liesenerbach & $1985-2013$ & 45.6 & $0.61^{b}$ & 0.3 & 0.65 & 12.6 \\
\hline Madatschbach & 1965-2013 & 11.4 & 0.6 & 0.3 & 0.65 & 3.1 \\
\hline Gsallbach & 1965-2013 & 15.3 & 0.58 & 0.3 & 0.65 & 4.0 \\
\hline Rostizbach & $1965-2013$ & 9.7 & 0.59 & 0.3 & 0.65 & 2.6 \\
\hline Mittertalbach & $1985-2013$ & 31.0 & $0.61^{b}$ & 0.3 & 0.65 & 8.5 \\
\hline Kraspesbach & 1993-2013 & 36.7 & $0.61^{b}$ & 0.3 & 0.65 & 10.1 \\
\hline Wazebach & $1965-2013$ & 17.9 & 0.62 & 0.3 & 0.65 & 5.0 \\
\hline Fissladbach & $1965-2013$ & 28.1 & 0.66 & 0.3 & 0.65 & 8.4 \\
\hline Verpeilbach & $1965-1993$ & 22.7 & 0.61 & 0.3 & 0.65 & 6.3 \\
\hline Längentalerbach & $1985-2013^{a}$ & 67.7 & $0.61^{b}$ & 0.3 & 0.65 & 18.6 \\
\hline Tscheybach & $1965-2013$ & 24.8 & 0.61 & 0.3 & 0.65 & 6.9 \\
\hline Gleirschbach & $1985-2013$ & 88.8 & $0.61^{b}$ & 0.3 & 0.65 & 24.5 \\
\hline Oberbergbach & $1992-2013$ & - & - & 0.3 & 0.65 & $18.2^{\mathrm{C}}$ \\
\hline Radurschlbach & $1965-2013$ & 34.6 & 0.68 & 0.3 & 0.65 & 10.7 \\
\hline Horlachbach & $1997-2013$ & 97.0 & $0.61^{b}$ & 0.3 & 0.65 & 26.7 \\
\hline Pitzbach & $1965-2013$ & $39.1 /-$ & $0.48 /-$ & 0.3 & 0.65 & $8.5 / 20.1^{c}$ \\
\hline
\end{tabular}

\begin{tabular}{|l|l|l|l|l|l|l}
\hline Bachname & $\mathrm{A}$ & $\mathrm{VG}$ & $\begin{array}{l}\text { Seehöhe } \\
(\text { Min-Max })\end{array}$ & $\mathrm{mN}$ & $\begin{array}{l}\text { Bachgefälle } \\
\text { Ga/Hg/Gn/Fs }\end{array}$ & Gd \\
\hline Schöntalbach & 2.8 & 0.00 & $1781-3000$ & 33 & $44 / 43 / 20 / 38$ & 1659 \\
\hline Schelfalmbach & 2.9 & 0.00 & $1960-3002$ & 30 & $23 / 23 / 12 / 18$ & 1758 \\
\hline Klammbach & 2.9 & 0.00 & $2052-2876$ & 32 & $14 / 10 / 13 / 4$ & 1151 \\
\hline Unterer Liesenerbach & 3.3 & 0.53 & $1978-3322$ & 28 & $37 / 41 / 17 / 22$ & 1903 \\
\hline Oberer Liesenerbach & 3.7 & 0.45 & $1983-3296$ & 33 & $41 / 49 / 18 / 36$ & 1472 \\
\hline Madatschbach & 3.8 & 0.12 & $1808-3438$ & 41 & $37 / 32 / 19 / 24$ & 1291 \\
\hline Gsallbach & 3.9 & 0.08 & $1828-3273$ & 43 & $21 / 25 / 22 / 18$ & 1118 \\
\hline Rostizbach & 4.8 & 0.01 & $1794-3392$ & 37 & $46 / 32 / 14 / 11$ & 1252 \\
\hline Mittertalbach & 5.1 & 0.02 & $1941-2996$ & 34 & $11 / 8 / 9 / 5$ & 1343 \\
\hline Kraspesbach & 6.3 & 0.07 & $1925-3055$ & 35 & $14 / 25 / 9 / 12$ & 1403 \\
\hline Wazebach & 6.7 & 0.09 & $1797-3528$ & 38 & $34 / 29 / 13 / 18$ & 1271 \\
\hline Fissladbach & 11.4 & 0.02 & $1798-3141$ & 32 & $20 / 16 / 5 / 7$ & 1613 \\
\hline Verpeilbach & 12.1 & 0.08 & $1805-3410$ & 40 & $16 / 17 / 6 / 15$ & 1373 \\
\hline Längentalerbach & 15.2 & 0.07 & $1954-3319$ & 37 & $15 / 8 / 4 / 4$ & 1363 \\
\hline Tscheybach & 16.4 & 0.00 & $1797-3056$ & 31 & $9 / 6 / 4 / 2$ & 1368 \\
\hline Gleirschbach & 16.7 & 0.07 & $1985-3188$ & 32 & $20 / 10 / 3 / 3$ & 1435 \\
\hline Oberbergbach & 23.7 & 0.28 & $1985-3457$ & 32 & $8 / 10 / 3 / 2$ & 1737 \\
\hline Radurschlbach & 24.0 & 0.01 & $1788-3352$ & 33 & $4 / 7 / 3 / 3$ & 1464 \\
\hline Horlachbach & 25.6 & 0.02 & $1914-3181$ & 32 & $9 / 10 / 2 / 6$ & 1373 \\
\hline Pitzbach & 27.2 & 0.46 & $1808-3549$ & 28 & $8 / 17 / 3 / 7$ & 1725 \\
\hline
\end{tabular}

dem Auslösen der automatischen Spü- Verhältnis von tatsächlichem (gemesleinrichtung gemessen (Tschada 1975). senen) Sedimentvolumen (gSV) und Die Form des Ablagerungskörpers er- jenem einer sohlparallelen Anlandung wies sich dabei als kegelförmig (Abb. 2), bis auf die Höhe der kritischen Auflast mit einer Verfeinerung des Sediments (theoretisches Ablagerungsvolumen, in Längsrichtung (Strobl 1980). Das tSV) wird als Skalierungsfaktor (sf) be- 

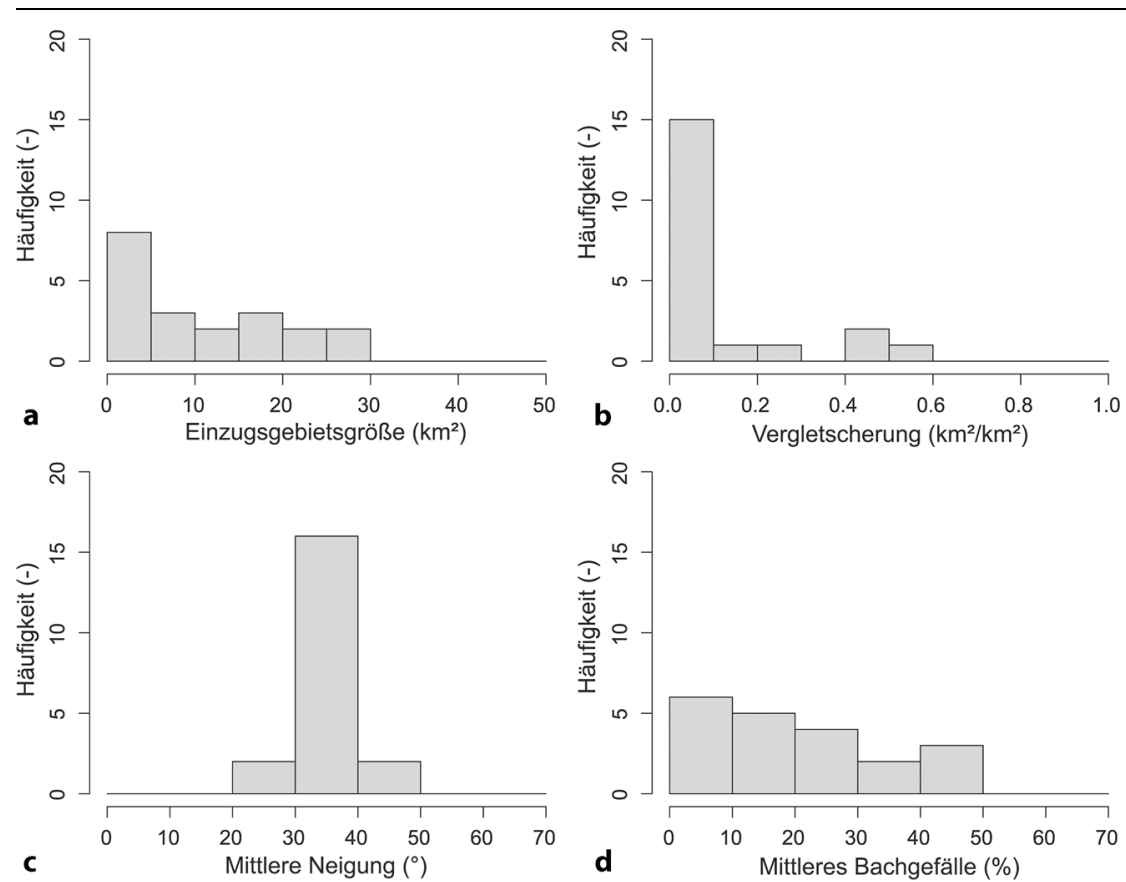

Abb. 3 Häufigkeitsverteilung von ausgewählten Gebietsparametern

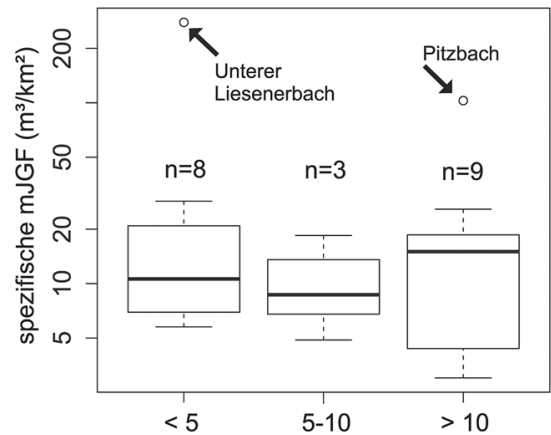

a

Einzugsgebietsgröße $\left(\mathrm{km}^{2}\right)$

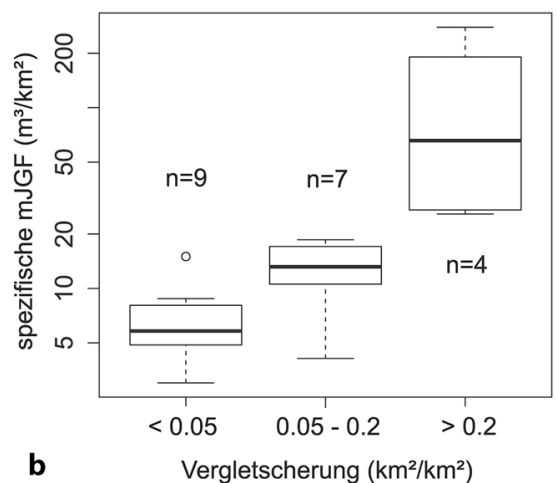

Abb. 4 Boxplot der spezifischen mJGF separiert nach Einzugsgebietsgröße und Vergletscherung mit der Anzahl an Untersuchungsgebiete je Klasse (n). Die Volumenangaben beziehen sich auf das reine Feststoffvolumen (ohne Porenanteile).

zeichnet und Tschada (1975) berichtet einen Wertebereich von 0.48 bis 0.66 für verschiedene Standorte.

Unter Annahme eines Grenzkorns zwischen Geschiebe und Schwebstoff von $1 \mathrm{~mm}$, entfallen rund $65 \%$ des gesamten Sediments auf das Geschiebe (ge) (Sommer und Lauffer 1982; Kammerlander 2017). Zusätzlich beträgt der Porenanteil $(e)$ rund $30 \%$. Das mittlere Geschiebevolumen einer Spülung (mGV) ist damit folgendermaßen definiert

$$
m G V=t S V \times s f \times g e \times(1-e)
$$

Zusammen mit der jährlichen Anzahl an Spülungen lässt sich damit ten in den Spülaufzeichnungen: zum einen aufgrund von möglichen Fehlzählungen, zum anderen durch manuelle Spülungen, die nicht automatisch bei „Vollfüllung“, sondern im Zuge von etwaigen Wartungsarbeiten ausgelöst wurden. Die Unsicherheiten der jeweiligen Parameter sind ausführlich in Kammerlander (2017) zusammengestellt und die relative Genauigkeit (Variationskoeffizient) der ermittelten Jahresgeschiebefrachten liegt bei rund $\pm 65 \%$.

\subsection{Kurzbeschreibung der untersuchten Einzugsgebiete}

Die 20 Wasserfassungen befinden in den Nordtiroler Zentralalpen und sie sind entweder Teil des Kaunertalkraftwerks oder der Kraftwerksgruppe Sellrain-Silz. Geologisch können alle Fassungsstandorte dem Kristallin zugeordnet werden und das Grundgestein besteht vor allem aus Gneis, Glimmerschiefer und Phyllit. Es handelt sich durchwegs um Hochgebirgsbäche, die am Standort der Wasserfassung ein hydrologisches Einzugsgebiet von $3 \mathrm{~km}^{2}$ bis $27 \mathrm{~km}^{2}$ aufweisen (Abb. 3a; Tab. 2). Die Wasserfassungen befinden sich auf einer Seehöhe zwischen $1.780 \mathrm{~m}$ und $2.050 \mathrm{~m}$, was im Bereich der aktuellen Waldgrenze liegt. Die Einzugsgebiete sind somit überwiegend der nivalen Klimazone zuzurechnen und die höchst gelegenen Bereiche sind teilweise vergletschert (Abb. 3b; Tab. 2). Die Einzugsgebiete unterscheiden sich hinsichtlich der Geländeform und der Topografie. Sie sind zwar alle durchwegs steil (Abb. 3c; Tab. 2), aber ihre Form reicht vom langgestreckten Trogtal bis zum kesselförmigen Kar.

Die Unterschiede der Topografie äußern sich auch am Längsprofil des Gewässernetzes. Das Bachgefälle ist ein sensibler Parameter in Bezug auf die Strömungskraft und die Transportkapazität von Geschiebe. Allerdings ist das Längsprofil von Hochgebirgsbächen in der Regel sehr ungleichmäßig und das Bachgefälle somit äußerst heterogen. Es stellt sich damit die Frage nach dem repräsentativen Längsgefälle eines ganzen Bachs.

In Bezug auf das Strömungsverhalten und die Strömungskraft unmittelbar oberhalb der Wasserfassung ist das lokale Bachgefälle maßgebend. Die lokale Strömungskraft hat allerdings kaum eine Aussagekraft auf die Jahresgeschiebefracht, denn je steiler der Bach, desto 

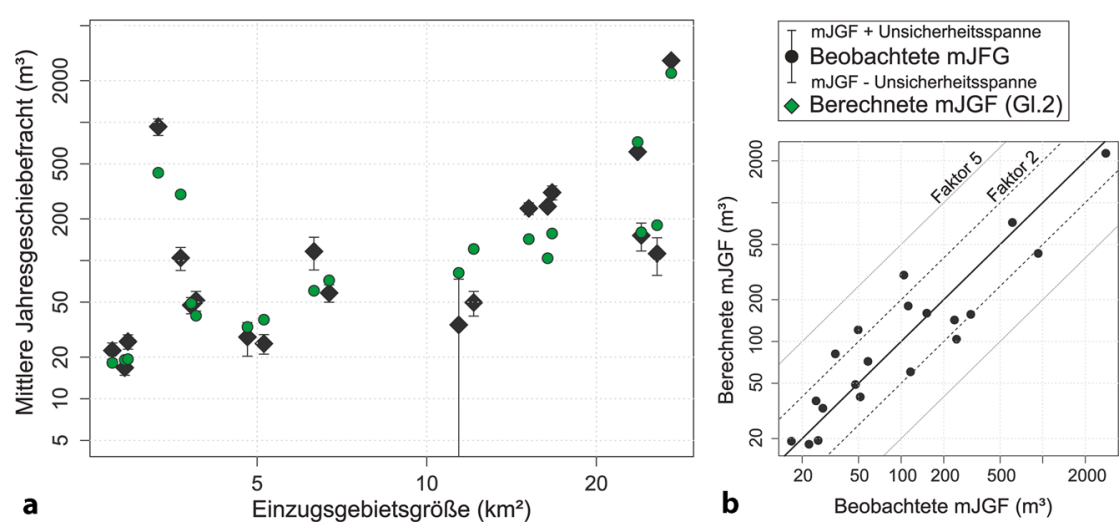

Abb. 5 a) Beobachtete und berechnete mJGF (Gleichung 2) nach EinzugsgebietsgröBeaufgetragen und b) Gegenüberstellung von berechneter und (mittlerer) beobachteter mJGF
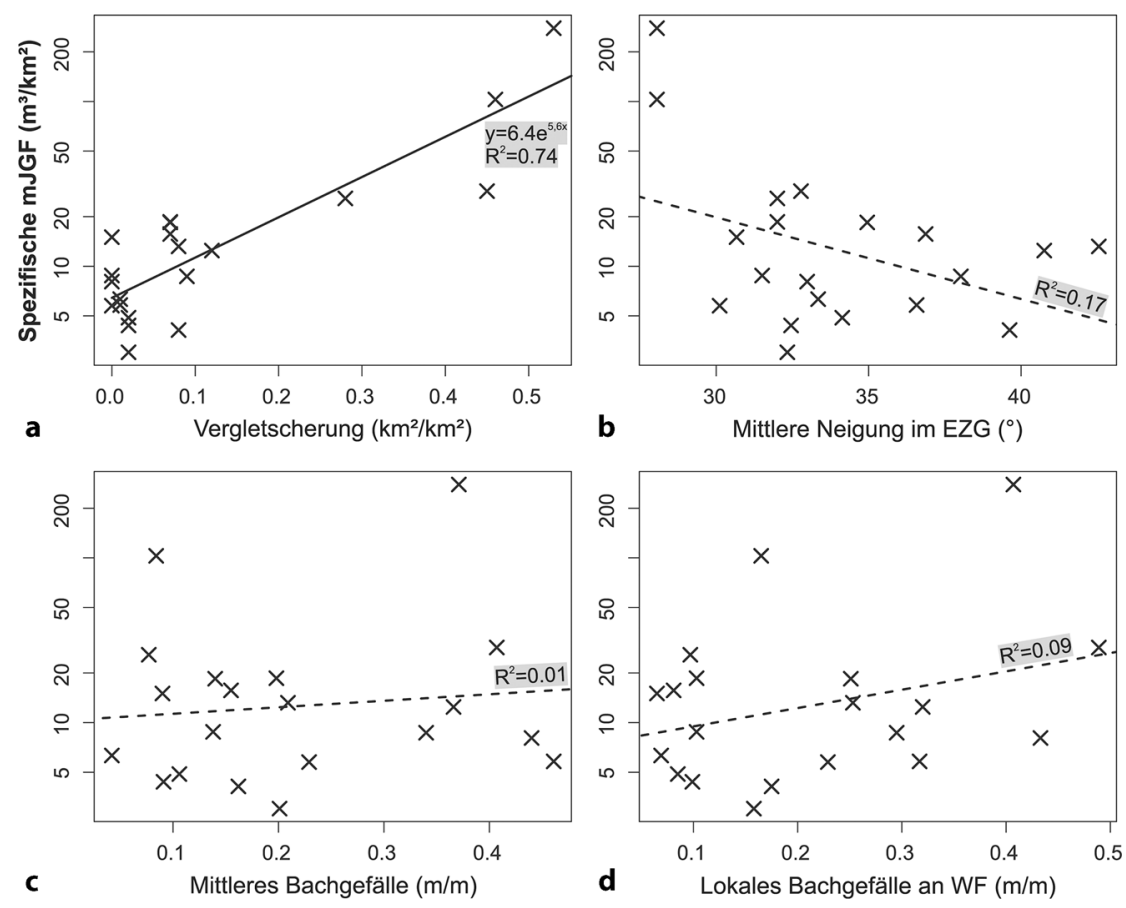

Abb. 6 Gegenüberstellung zwischen mJGF (in logarithmischer Skalierung) und a) der Vergletscherung, b) der mittleren Neigung im Einzugsgebiet, c) dem mittleren Bachgefälle des Hauptgerinnes ( $\mathrm{Hg}$ in Tab. 2) und d) das lokale Bachgefälle bachaufwärts der Wasserfassung (Ga in Tab. 2)

gröber in der Regel die Kornverteilung und desto höher auch die Stabilität der Sohle. Es wurde daher das mittlere Bachgefälle und dessen räumliche Variabilität in unterschiedlichen räumlichen Skalen bestimmt (Tab. 2). Die kleinste räumliche Skala umfasst den Gerinnneabschnitt direkt oberhalb einer Wasserfassung (Ga in Tab. 2). Die mittlere Skala entspricht dem Hauptgerinne (Abb. 3d; Hg in Tab. 2), welches aus Gründen der Vergleichbarkeit ein-

\subsection{Ergebnisse}

\subsubsection{Mittlere Jahresgeschiebefracht}

Der arithmetische Mittelwert der Jahresgeschiebefrachten (mJGF) unterscheidet sich stark zwischen den einzelnen Gebieten, mit einem Minimum von $17 \mathrm{~m}^{3}$ (Fissladbach) und einem Maximum von $2.791 \mathrm{~m}^{3}$ (Pitzbach). Die hohe Variabilität bestätigt sich auch in Bezug auf die mittleren spezifischen Jahresgeschiebefrachten (Geschiebefracht je $\mathrm{km}^{2}$ Einzugsgebietsfläche; mJGF/A), welche zwischen $3.0 \mathrm{~m}^{3} / \mathrm{km}^{2}$ (Fissladbach) und $278.5 \mathrm{~m}^{3} / \mathrm{km}^{2}$ (Unterer Liesenerbach) liegen.

Ein statistischer Zusammenhang zwischen mJGF und den topografischen Gebietsparametern (Tab. 2) wurde mittels Korrelations- und Regressionsanalysen getestet. Die mittlere Jahresgeschiebefracht korreliert höchst signifikant mit der Einzugsgebietsfläche (A) und dem Vergletscherungsgrad (VG). Der Einfluss der Gebietsgröße ist dabei nahezu proportional (Abb. 4a), jener des Vergletscherungsgrades exponentiell (Abb. 4b).

Die multiple Regressionsanalyse ergibt folgenden empirischen Zusammenhang:

$$
\begin{gathered}
m J G F=6.63 \times A^{0.99} \times \exp (5.65 V G) \\
\left(R^{2}=0.84\right)
\end{gathered}
$$

Hierbei bezieht sich mJGF auf das Feststoffvolumen an Geschiebe in $\mathrm{m}^{3}$, A auf die Einzugsgebietsgröße in $\mathrm{km}^{2}$ und VG auf das Verhältnis von Gletscherfläche zu Gesamtfläche $\left(\mathrm{km}^{2} / \mathrm{km}^{2}\right)$. Diese beiden Gebietsparameter erklären $84 \%$ der Streuung von mJGF, die Güte der Regressionsgleichung ist in Abb. 5 dargestellt.

Für die Umrechnung in das Ablagerungsvolumen ist das Ergebnis von Gleichung 2 durch $(1-e)=0.7$ zu dividieren, für die Umrechnung in Tonnen ist es mit 2.65 to $/ \mathrm{m}^{3} \mathrm{zu}$ multiplizieren.

Im Vergleich zur Einzugsgebietsfläche und dem Vergletscherungsgrad, zeigen die Korrelationsanalysen, dass die restlichen topografischen Parameter (bspw. mittlere Hangneigung, Bachgefälle oder Einzugsgebietsform) keinen wesentlichen Einfluss auf mJGF haben (Abb. 6b bis 6d). Der grundsätzlich starke Einfluss des Bachgefälles auf die Transportkapazität in einem Gerinne spiegelt sich somit nicht in der mittleren Jahresgeschiebefracht wider. 
Tab. 3 Statistische Kenngrößen zur Verteilung der Jahresgeschiebefrachten an den 20 Wasserfassungen mit der Anzahl an Beobachtungsjahren (N), der mittleren Jahresgeschiebefracht (mJGF) und der Standardabweichung der Jahresgeschiebefrachten (sd(JGF)). Die Volumenwerte beziehen sich auf das reine Feststoffvolumen (ohne Poren). Für die Umrechnung in das Lagerungsvolumen sind die Werte mit 0.7 zu dividieren, für Massenangaben (to) mit 2.65 to $/ \mathrm{m}^{3} \mathrm{zu}$ multiplizieren.

\subsubsection{Zeitliche Variabilität der Jahresgeschiebefrachten}

Die Jahresgeschiebefrachten unterscheiden sich nicht nur räumlich (nach Standorten der Wasserfassung), sondern auch zeitlich. Die Spülaufzeichnungen geben Rückschluss auf die Jahresgeschiebefrachten von bis zu 49 Jahren und erlauben somit eine Analyse von deren Verteilung (Abb. 7). Einige Kennzahlen der empirischen Verteilungen der Jahresgeschiebefrachten sind in Tab. 3 zusammengestellt.

An allen Wasserfassungen weisen die Jahresgeschiebefrachten eine rechtsschiefe Verteilung auf. Dies bedeutet, dass Jahre mit Geschiebefrachten kleiner als der mJGF häufiger auftreten als Jahre mit größeren Frachten. Es ergeben sich somit für wenige Jahre äußerst große Geschiebefrachten (Abb. 7b). Es ist hierbei festzuhalten, dass die Aussagekraft der Daten auf hydrologische Normaljahre ohne (seltene) Extremereignisse beschränkt ist. Während solcher Extremereignisse ist ein Ausfallen der Spüleinrichtung (bspw. infolge einer Komplettverlegung der Wasserfassung) nicht ausgeschlossen. Entsprechend den Aufzeichnungen der TIWAG sind solche Ereignisse die seltene Ausnahme. Sie beeinflussen somit nicht die Aussagekraft der mittleren jährlichen

\begin{tabular}{|l|l|l|l|l|l|l|l}
\hline Bachname & N & mJGF & sd(JGF) & Median & $\begin{array}{l}25 \% \text { Quan- } \\
\text { til }\end{array}$ & $\begin{array}{l}75 \% \\
\text { Quantil }\end{array}$ & Schiefe \\
\hline Schöntalbach & $(-)$ & $\left(\mathrm{m}^{3}\right)$ & $\left(\mathrm{m}^{3}\right)$ & $\left(\mathrm{m}^{3}\right)$ & $\left(\mathrm{m}^{3}\right)$ & $\left(\mathrm{m}^{3}\right)$ & $(-)$ \\
\hline Schelfalmbach & 27 & 22 & 16 & 18 & 9 & 32 & 1.3 \\
\hline Klammbach & 29 & 17 & 14 & 10 & 6 & 22 & 1.8 \\
\hline Unterer Liesener- & 29 & 26 & 23 & 18 & 12 & 28 & 2.0 \\
\hline bach & 930 & 958 & 538 & 296 & 1349 & 1.6 \\
\hline Oberer Liesenerbach & 29 & 104 & 122 & 54 & 44 & 119 & 2.8 \\
\hline Madatschbach & 49 & 48 & 52 & 37 & 9 & 64 & 2.3 \\
\hline Gsallbach & 49 & 51 & 76 & 22 & 8 & 57 & 2.9 \\
\hline Rostizbach & 49 & 28 & 80 & 5 & 3 & 16 & 4.7 \\
\hline Mittertalbach & 29 & 25 & 26 & 17 & 9 & 26 & 2.5 \\
\hline Kraspesbach & 29 & 116 & 216 & 64 & 38 & 101 & 4.6 \\
\hline Wazebach & 49 & 58 & 70 & 39 & 20 & 76 & 3.1 \\
\hline Fissladbach & 49 & 34 & 84 & 8 & 2 & 25 & 4.8 \\
\hline Verpeilbach & 29 & 50 & 69 & 25 & 13 & 57 & 2.6 \\
\hline Längentalerbach & 28 & 238 & 232 & 141 & 67 & 340 & 1.2 \\
\hline Tscheybach & 49 & 246 & 208 & 189 & 100 & 296 & 1.6 \\
\hline Gleirschbach & 28 & 310 & 236 & 251 & 170 & 353 & 1.7 \\
\hline Oberbergbach & 22 & 662 & 377 & 587 & 409 & 862 & 0.6 \\
\hline Radurschlbach & 49 & 152 & 357 & 43 & 16 & 139 & 5.6 \\
\hline Horlachbach & 17 & 112 & 188 & 61 & 48 & 93 & 3.8 \\
\hline Pitzbach & 49 & 2791 & 2345 & 2024 & 1102 & 3621 & 1.6 \\
\hline
\end{tabular}

Geschiebefracht, wohl aber jene einzelner, seltener Extremereignisse.

An den meisten Wasserfassungen kann die beobachtete Verteilung von JGF gut mit der lognormalen Wahrscheinlichkeitsfunktion angenähert werden (Abb. 9a). Die logarithmische Normalverteilung ist eine Wahrscheinlichkeitsfunktion mit den zwei Parametern $\mu$ und $\sigma$. Geht man nun davon aus, dass die Jahresgeschiebefrachten eines Baches lognormalverteilt sind und somit JGF als lognormalverteilte Zufallsvariable $\mathrm{x}$ definiert werden kann, ergibt sich die Dichtefunktion mit:

$$
\begin{aligned}
& f(x)=\frac{1}{x \sigma \sqrt{2 \pi}} \exp \left[-\frac{(\ln (x)-\mu)^{2}}{2 \sigma^{2}}\right] \\
& \quad \text { für } x>0
\end{aligned}
$$

Für empirische Verteilungen wie jene der JGF lassen sich die beiden Parameter $\mu$ und $\sigma$ durch den Mittelwert und die Standardabweichung der logarithmierten JGF annähern. In Analogie zu den Korrelations- und Regressionsanalysen mit mJGF wurden auch für diese statistischen Kenngrößen nach Abhängigkeiten zu Gebietsparametern gesucht (Abb. 8).

Demnach korreliert $\mu$ höchst signifikant mit der Einzugsgebietsgröße (A) und der Vergletscherung (VG). Die multiple Regressionsanalyse führt dabei zu folgendem empirischen Zusammenhang

$$
\begin{gathered}
\widehat{\mu}=1.27+0.96 \ln (A)+6.22 V G \\
\mathrm{R}^{2}=0.76
\end{gathered}
$$

Hierin ist A wiederum in $\mathrm{km}^{2}$ und VG in $\mathrm{km}^{2} / \mathrm{km}^{2}$ zu verwenden.

Im Gegensatz dazu korreliert $\sigma$ signifikant mit der mittleren Neigung im Einzugsgebiet $(\mathrm{mN})$. Im Vergleich $\mathrm{zu}$ $\mu$ ist der Zusammenhang allerdings schwächer und die lineare Regressionsgleichung erklärt lediglich $26 \%$ der Varianz. Die grafische Gegenüberstellung von $\mathrm{mN}$ und $\sigma$ zeigt drei Ausreißer, mit deutlich höheren $\sigma$-Werten als die übrigen (Abb. 8b). Werden diese Ausreißer in der Regressionsanalyse nicht berücksichtigt, ergibt sich ein deutlich besseres Bestimmtheitsmaß von $\mathrm{R}^{2}=0.54$ (Abb. 8b). Die empirische Gleichung lautet

$$
\begin{gathered}
\widehat{\sigma}=-0.046+0.031 \mathrm{mN} \\
\mathrm{R}^{2}=0.54
\end{gathered}
$$

wobei mN mit der Einheit Altgrad $\left(^{\circ}\right)$ definiert ist.

Mit den Gleichungen 4 und 5 lassen sich somit die Parameter der Lognormalverteilung schätzen, welche sich ihrerseits für die empirische Häufigkeitsverteilung von Jahresgeschiebefrachten in Hochgebirgsbächen eignet. Es 

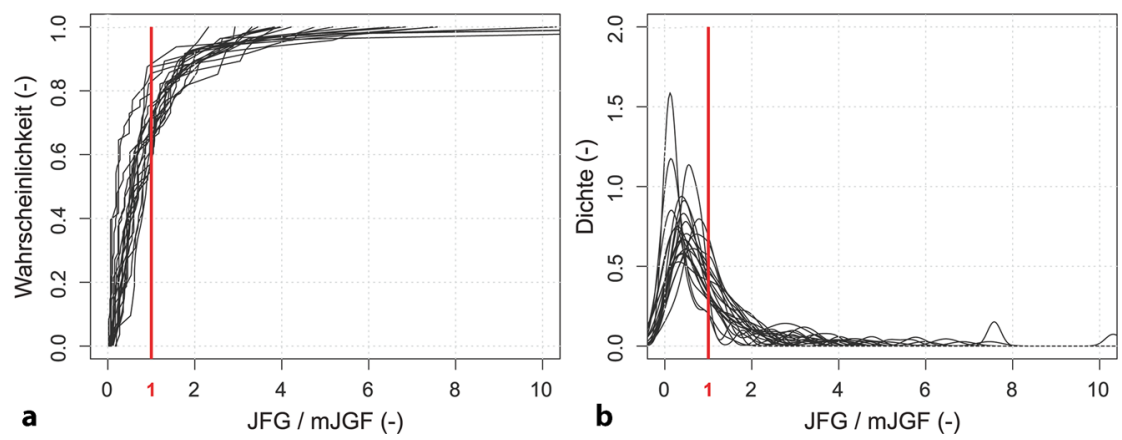

Abb. 7 a) Kumulierte Verteilungsfunktionen der JGF aller 20 Einzugsgebiete und b) geglättete Histogramme (Kern-Dichte-Schätzung)
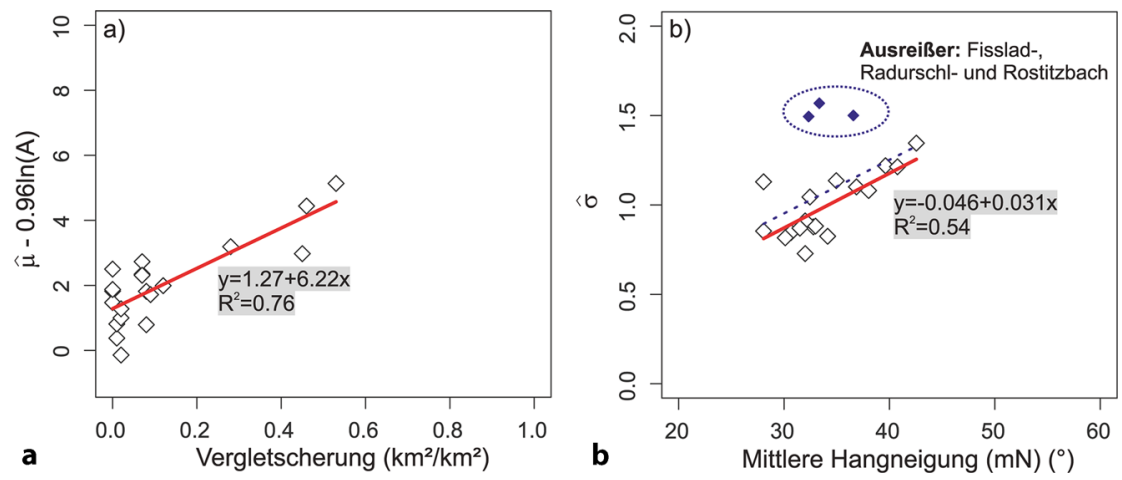

Abb. 8 Korrelation und Regression der empirischen Parameter der logarithmischen Normalverteilung mit topografischen Gebietsparametern entsprechend den Gleichungen (4) und (5).

können mit dieser „geschätzten“ Lognormalverteilung etwaige statistische Kenngrößen der Jahresgeschiebefrachten (bspw. Quantilwerte, Standardabweichung) errechnet werden. Die Güte dieser Methode ist in Abb. 9b dargestellt. Im Vergleich zu der angepassten Lognormalverteilung ist die Spannweite der Ungenauigkeit deutlich größer. Für die meisten Gebiete liegt das Verhältnis von berechneten und beobachteten Quantilwerten innerhalb eines Faktors von 2 (Abb. 9b).

\section{Variabilität der Transporteffizienz}

Eine wesentliche Erkenntnis der obigen Ergebnisse ist der statistische Zusammenhang zwischen mJGF und den Gebietsparametern A und VG. Dieser lässt sich in gewisser Weise auch durch rein theoretische Überlegungen interpretieren.

Die Geschiebefracht nimmt zum einen annähernd linear mit der Einzugsgebietsgröße zu. Im Gegensatz würde eine nichtlineare Abhängigkeit zwischen mJGF und A ein Ungleichgewicht bedeuten, was zwangsläufig zu einem Nettorückhalt (bei einem Exponenten kleiner 1) oder -austrag (bei einem Exponenten größer 1) führt. Dies ist in den untersuchten Gebieten allerdings nicht der Fall.

Im Unterschied dazu ist der $\mathrm{Zu}$ sammenhang zwischen mJGF und der Vergletscherung im Einzugsgebiet exponentiell und somit viel stärker. Die möglichen Ursachen für diese starke Abhängigkeit sind entweder das erhöhte Abflussvermögen in vergletscherten Gebieten infolge des Gletscherrückgangs oder aber das hohe Potenzial an feinem, leicht mobilisierbarem Sediment im Vorfeld der Gletscher.

Es stellt sich ferner die grundsätzliche Frage, ob die jährliche Transportleistung eines Hochgebirgsbachs eine reine Funktion des Jahresabflusses ist, oder diese durch zusätzliche Faktoren wie einer jahresweise unterschiedlichen Verfügbarkeit von feinem Sediment beeinflusst wird. Eine Möglichkeit, um diese Frage $\mathrm{zu}$ beantworten, ist das Verhältnis zwischen einer theoretisch errechneten „abflussinduzierten Transportleistung" und der gemessenen Jahresgeschiebefracht. Ist nun in einer bestimmten Bachstrecke der Geschiebetransport eine alleinige Funktion des Abflusses, sollte das Verhältnis dieser beiden Werte zeitlich annähernd konstant sein. Andernfalls ist dieser eindeutige Zusammenhang nicht gegeben und die Transporteffizienz zeitlich variabel.

\subsection{Ermittlung der Transporteffizienz}

In einer bestimmten Bachstrecke nimmt der Geschiebetransport in der Regel mit dem Abfluss zu. Aktuelle Untersuchungen zeigen, dass die Zunahme bei geringen Abflüssen höchst nichtlinear mit Exponenten $>1$, die absolute Transportrate allerdings sehr gering ist. Bei hohen Abflüssen hingegen ist die Zunahme mit dem Abfluss schwächer, dafür kann die absolute Menge an transportiertem Geschiebe sehr groß sein.

Unter der Annahme einer eindeutigen Funktion zwischen Abfluss und Geschiebetransport lässt sich, unter Kenntnis der Abflussganglinie, auch die Geschiebefracht berechnen. Allerdings ist der Zusammenhang zwischen Abfluss und Geschiebetransport stark gebietsabhängig und variiert im Wesentlichen je nach Kornzusammensetzung des Sohlmaterials, dem Bachgefälle, der Gerinnegeometrie und weiterer, nicht näher quantifizierbarer Parameter wie bspw. Verfügbarkeit an feinem Geschiebe.

In einer aktuellen Studie präsentierten Schneider et al. (2015b) eine Geschiebetransportfunktion, welche auf Grundlage zahlreicher Feldmessdaten entwickelt wurde. Unter diversen Vereinfachungen (siehe Kammerlander 2017) lässt sich diese Funktion in folgende Form überführen:

$$
Q_{\mathrm{b}}=K \times\left\{\begin{array}{l}
0.002 \times\left(\frac{Q}{Q_{\mathrm{r}}}\right)^{6.44} \times Q^{0.6} \\
\text { für } Q / Q_{\mathrm{r}} \leq 1.58 \\
14 \times\left(1-\frac{0.831}{\left(Q / Q_{\mathrm{r}}\right)^{0.28}}\right)^{4.5} \times Q^{0.6} \\
\text { für } Q / Q_{\mathrm{r}}>1.58
\end{array}\right.
$$

Hierin ist $\mathrm{Q}_{\mathrm{b}}$ die Geschiebetransportrate $\left(\mathrm{m}^{3} / \mathrm{s}\right)$, K die Transporteffizienz $\left(\mathrm{m}^{1.2} / \mathrm{s}^{0.4}\right)$, $\mathrm{Q}$ der Abfluss und $\mathrm{Q}_{\mathrm{r}}$ der kritische Abfluss für den $\mathrm{D}_{50}$ des Sohlmaterials (beide $\mathrm{m}^{3} / \mathrm{s}$ ). Diese Transportformel berücksichtigt den unterschiedlichen Zusammenhang zwischen Geschiebetransport und Abfluss bei kleinen $\left(\mathrm{Q} / \mathrm{Q}_{\mathrm{r}}<1.58\right)$ und großen Abflüssen $\left(\mathrm{Q} / \mathrm{Q}_{\mathrm{r}}>1\right.$ 1.58). Der Vergleich mit unabhängigen Felddaten bestätigte 


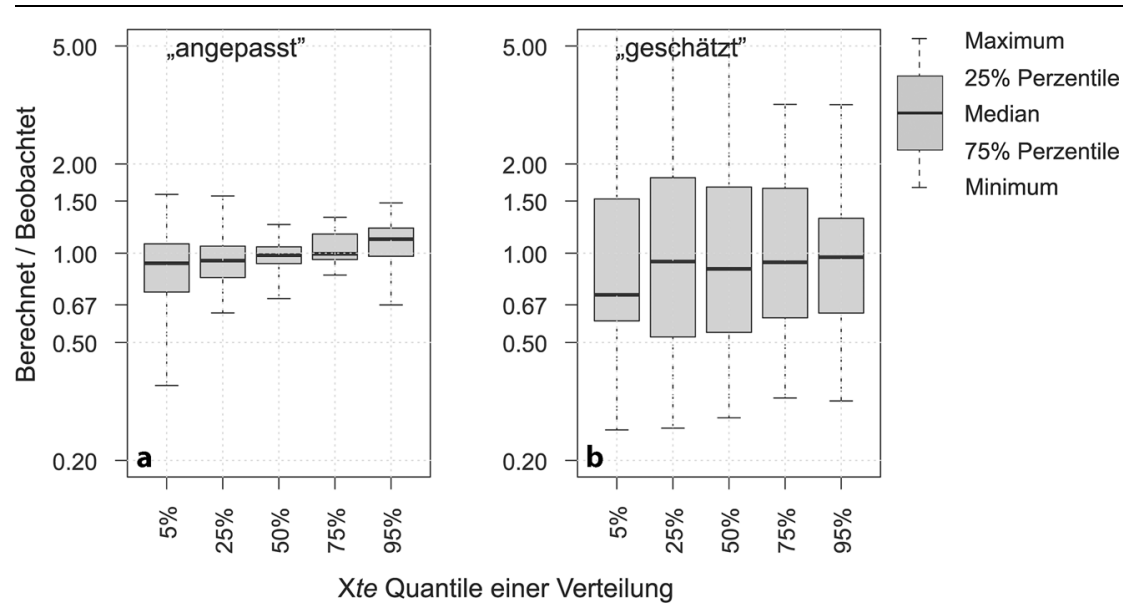

Abb. 9 Boxplot des Verhältnisses von beobachtetem Quantilwert und mittels theoretischer Verteilungsfunktion berechnetem Quantilwert. Die theoretische Verteilungsfunktion basiert auf a) den angepassten Parametern und b) den mittels Gleichung 4 und 5 geschätzten Parametern; jeder Boxplot enthält die Daten aller 20 Einzugsgebiete
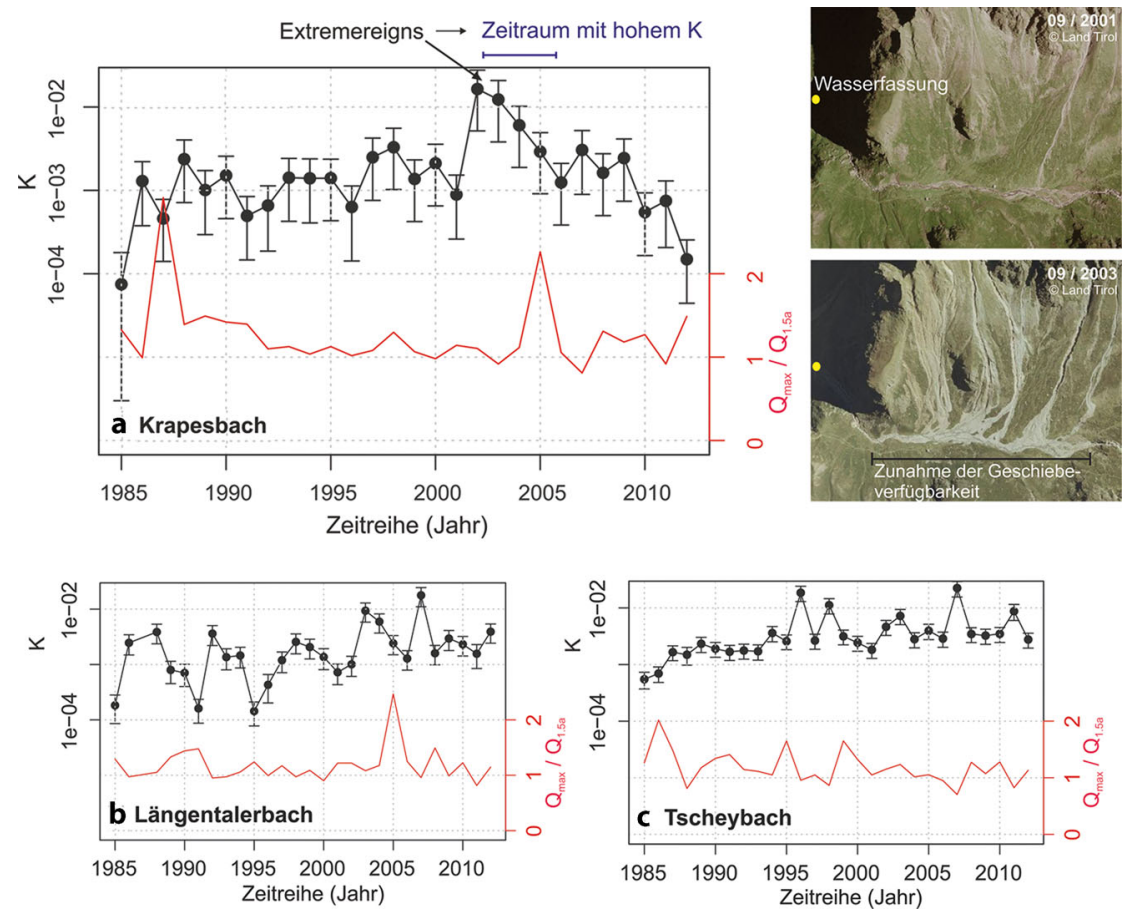

Abb. 10 Jahresreihe der Transporteffizienz K (schwarze Punkte mit Ungenauigkeitsspanne) und des Jahreshöchstabflusses (standardisiert mit Q ${ }_{1.5 a}$ ) im a) Kraspesbach (mit einem Extremereignis im Jahr 2002), b) Längentalerbach und c) Tscheybach

dabei, dass Gleichung 6 den Anstieg der Transportrate mit dem Abfluss gut wiedergibt (Kammerlander 2017). Das Grenzkriterium $\mathrm{Q} / \mathrm{Q}_{\mathrm{r}}=1.58$ bzw. der Übergang zwischen schwachem Transport von Feinmaterial und starkem Transport von grobem Sohlmaterial wird in steilen Gebirgsbächen nur selten überschritten. In dieser Untersuchung wird der Übergang in Abhän- wurden jahresweise getrennt und der rechte Term von Gleichung 6 wurde mit jedem Abflusswert dieser Jahresreihe berechnet, über das Messintervall von 15 min integriert und aufsummiert:

$J G F=$

$$
K \times \sum\left\{\begin{array}{l}
0.002 \times\left(\frac{Q}{0.5 Q_{1.5 a}}\right)^{6.44} \times Q^{0.6} \times d t \\
\text { für } \frac{Q}{0.5 Q_{1.5 a}} \leq 1.58 \\
14 \times\left(1-\frac{0.831}{\left(\frac{Q}{0.5 Q_{1.5 a}}\right)^{0.28}}\right)^{4.5} \times Q^{0.6} \times d t \\
\text { für } \frac{Q}{0.5 Q_{1.5 \mathrm{a}}}>1.58
\end{array}\right.
$$

Der rechte Term von Gleichung 7 wird in weiterer Folge als ,abflussinduzierte Transportkapazität" bezeichnet. Zumal die Jahresgeschiebefracht bekannt ist, lässt sich die tatsächliche Transporteffizienz K wie folgt bestimmen:

$$
K=\frac{J G F}{\sum f\left(\frac{Q}{0.5 Q_{1.5 \mathrm{a}}}\right)}
$$

Die Hypothese eines konstanten, mit der Transportgleichung beschreibbaren Zusammenhangs zwischen Geschiebetransport und Abfluss ist dann gegeben, wenn sich die Transporteffizienz $\mathrm{K}$ an einer Wasserfassung während der Jahre nicht ändert. Die Ergebnisse dieser Berechnung zeigen allerdings ein anderes Bild: Die Transporteffizienz K schwankt im Mittel um zwei Größenordnungen. Diese Variabilität ist nicht auf den Abfluss oder Unsicherheiten in den Messungen zurückzuführen, sondern auf die verbleibenden Einflussfaktoren, wie eben Gerinnegeometrie, Fließrauheit oder Verfügbarkeit von feinem Geschiebe. Die ersten beiden Einflussfaktoren ändern sich in Hochgebirgsbächen in der Regel kaum mit der Zeit. Im Gegensatz dazu kann sich die Verfügbarkeit von transportierbarem Geschiebe sehr wohl ändern. Zum einen führen größere Hochwässer zu einem Aufbrechen der Deckschicht und der damit einhergehenden Freisetzung von feinem Sohlmaterial aus der Unterschicht (Yager et al. 2012; Gintz et al. 1996). Zum anderen unterscheidet sich der Geschiebeeintrag in ein Gewässer auch in der Mikroskala, bspw. je nach dem Abflussursprung (Kammerlander 2017; Mao et al. 2014).

Die Auswirkung eines Extremereignisses auf die mittelfristige Transporteffizienz konnte an einem der untersuchten Einzugsgebiete quantifiziert wer- 


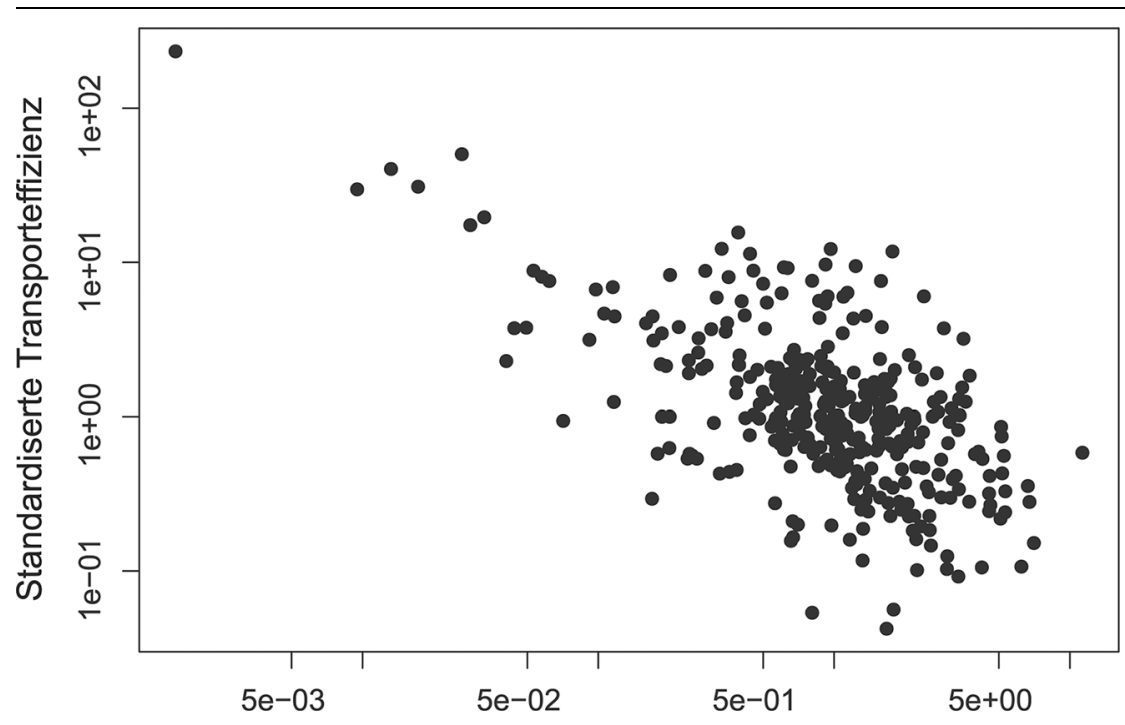

Standardisierte „abflussinduzierte Transportkapazität”

Abb. 11 Gegenüberstellung zwischen der jährlichen, abflussinduzierten Transportkapazität und der Transporteffizienz. Beide Variablen wurden mit deren gebietsspezifischen Mittelwerten standardisiert

den. Am Kraspesbach im Sellraintal ereignete sich im Jahr 2002 ein Starkregenereignis, welches $\mathrm{zu}$ intensiven Sedimenteinstößen aus den angrenzenden Steilhängen führte (Abb. 10). Während dieses Ereignisses wurde die Wasserfassung komplett überschottert, die Abfluss- oder Geschiebefracht ist somit nicht bekannt. Allerdings nimmt die Transporteffizienz nach dem Ereignis um rund eine Größenordnung zu und geht innerhalb der darauffolgenden drei Jahre auf das Ursprungsniveau zurück (Abb. 10). Die hohe Materialverfügbarkeit durch den Seiteneinstoß führte somit zu einer rund 10-mal höheren Transporteffizienz im Hauptgerinne.

Allerdings schwankt die jährliche Transportkapazität auch ohne Extremereignis beträchtlich. In Abb. $10 \mathrm{~b}$ ist die Jahresreihe des Längentalerbachs dargestellt. Sie deutet auf einen periodischen Wechsel niedriger und hoher Transporteffizienz. Eine Abhängigkeit vom Jahreshöchstabfluss lässt sich dabei nicht erkennen. Als Grund dieser periodischen Zyklen von $\mathrm{K}$ wird vielmehr der episodische Materialeintrag aus einer steilen Runse vermutet. Die Transporteffizienz des Tscheybachs (Abb. 10c) ist hingegen verhältnismäßig konstant und weist nur einzelne Spitzen auf. Aber auch hier ist keine Abhängigkeit vom Jahreshöchstabfluss zu erkennen.
Die Ergebnisse zeigen ferner, dass $\mathrm{K}$ in Jahren mit geringer "abflussinduzierter Transportkapazität" mehrheitlich höher ist als in Jahren mit viel Abfluss (Abb. 11). Das bedeutet, dass in "trockenen“ Jahren verhältnismäßig mehr Geschiebe transportiert wird als in feuchten Jahren; hierbei ist die nichtlineare Abhängigkeit zwischen Abfluss und Geschiebetransport explizit berücksichtigt (Gleichung 7). Insgesamt ist dadurch die Variabilität der JGF häufig kleiner, als es ein starrer Zusammenhang zwischen Abfluss und Geschiebetransport vermuten ließe.

Eine mögliche Erklärung ist wiederum eine beschränkte Geschiebeverfügbarkeit, die in feuchten Jahren einen vermehrten Transport an Geschiebe verhindert bzw. die Effizienz verringert. Im Gegenzug ist anzunehmen, dass der „regelmäßige“ Eintrag aus etwaigen Sedimentquellen auch in noch so trockenen Jahren transportiert werden kann. Insgesamt verdeutlichen die Ergebnisse, dass die Menge an transportiertem Geschiebe in der überwiegenden Zeit nicht durch die abflussinduzierte Transportkapazität, sondern durch andere Mechanismen, wie Verfügbarkeit von feinem Geschiebe, reguliert wird. fluss der Vergletscherung auf die mittlere Jahresgeschiebefracht (Kapitel 2.4). Im Gletschervorfeld ist in der Regel ein hohes Potenzial an feinem (transpor-
Dies erklärt auch den starken Ein- tierbarem) Geschiebe vorhanden, weshalb in stark vergletscherten Gebieten auch eine deutlich höhere Transporteffizienz vorherrscht. Der Einfluss der Vergletscherung auf die mittlere Jahresgeschiebefracht ist demnach weniger dem erhöhtem Abfluss infolge des Gletscherrückgangs geschuldet, sondern vielmehr der starken Sedimentbelieferung in das Gerinne.

\section{Schlussfolgerung}

Die mittlere Jahresgeschiebefracht (mJGF) der untersuchten Hochgebirgsbäche korreliert höchst signifikant mit der Einzugsgebietsgröße (A) und der Vergletscherung (VG). Der Zusammenhang zwischen mJFG und A ist dabei linear und die mittleren spezifischen Jahresfrachten $\left(\mathrm{m}^{3} / \mathrm{km}^{2}\right)$ sind somit unabhängig von Einzugsgebietsgröße.

Der Einfluss der Vergletscherung ist dagegen exponentiell und begründet sich maßgeblich durch die hohe Geschiebeverfügbarkeit in den Gletschergebieten. Die Geschiebeverfügbarkeit kann sich allerdings auch aufgrund anderer Einflussfaktoren, wie bspw. der Sedimentbelieferung aus den Grabeneinhängen oder seitlichen Zubringern/ Runsen, unterscheiden. Einen entscheidenden Faktor haben hier wahrscheinlich die geologischen Rahmenbedingungen. Diesbezüglich weisen die 20 Untersuchungsgebiete eine hohe Ähnlichkeit auf. Es handelt sich durchwegs um Hochgebirgsbäche der kristallinen Nordtiroler Zentralalpen. Die ermittelten Regressionsgleichungen sind deshalb nur für Hochgebirgsbäche mit vergleichbaren Rahmenbedingungen geeignet.

Die Langzeitdaten der Jahresgeschiebefrachten zeigen auch eine hohe zeitliche Variabilität. Erstaunlicherweise erklärt sich diese nicht nur durch die von Jahr zu Jahr unterschiedlichen Abflussverhältnisse. Vielmehr ändert sich auch die Transporteffizienz, wobei in trockenen Jahren verhältnismäßig mehr Geschiebe transportiert wird als in feuchten. Die Jahresgeschiebefracht wird demnach weniger durch den Abfluss, sondern durch die limitierte Verfügbarkeit von feinem Geschiebe begrenzt. Würde der Eintrag von transportierbarem Geschiebe künstlich erhöht werden, ist es durchaus möglich, dass auch diese Fracht durch den natürlichen Abfluss weitertransportiert werden kann (Kammerlander et al. 2017). Im Umkehrschluss stimmen die- 
se Ergebnisse auch mit den Erfahrungen an den alpinen Wasserfassungen überein. Hier ist der Geschiebeeintrag zwar unverändert, der Abfluss im Unterwasser der Anlage allerdings stark reduziert. Es konnte aber bereits in einer früheren Studie gezeigt werden, dass im Unterwasser der Fassungen keine Anlandungstendenzen bestehen (Schönlaub und Hofer 2009). Demnach besitzen sedimentlimitierte, steile Hochgebirgsbäche eine Anpassungsfähigkeit hinsichtlich der Veränderung des übergeordneten Geschiebehaushalts. Es ist die Aufgabe zukünftiger
Untersuchungen, diese Anpassungsfähigkeit näher einzugrenzen, nicht zuletzt um die Geschiebedurchgängigkeit auch in Zukunft zu gewährleisten.

Danksagung Die Autoren danken der TIWAG für die Bereitstellung ihrer Langzeitaufzeichnungen und der Erlaubnis zu zusätzlichen Messungen an einigen ihrer Anlagen. Die Untersuchungen sind Teil des Forschungsprojekts DevoBeTA-CC (B286218), welches durch das Förderprogramm Austrian Climate Research Programme (ACRP) des Klimaund Energiefonds finanziert ist.
Open access funding provided by University of Innsbruck and Medical University of Innsbruck.

Open Access Dieser Artikel wird unter der Creative Commons Namensnennung 4.0 International Lizenz (http:// creativecommons.org/licenses/by/4. 0/deed.de) veröffentlicht, welche die Nutzung, Vervielfältigung, Bearbeitung, Verbreitung und Wiedergabe in jeglichem Medium und Format erlaubt, sofern Sie den/die ursprünglichen Autor(en) und die Quelle ordnungsgemäß nennen, einen Link zur Creative Commons Lizenz beifügen und angeben, ob Änderungen vorgenommen wurden.
Bunte, K., Abt, S., Potyondy, J. P., Ryan, S. E. (2004): Measurement of coarse gravel and cobble transport using portable bedload traps, J Hydraul Eng 130(9): 879-893 doi:10.1061/ (ASCE) 0733-9429(2004) 130:9(879)

Gintz, D., Hassan, M. A., Schmidt, K.-H. (1996): Frequency and magnitude of bed load transport in a mountain river. Earth Surf Proc Land 21:433445

Habersack, H., Wagner, B., Schoder, A., Hauer C. (2013): Die Bedeutung von Feststoffhaushalt und Sedimentdurchgängigkeit für eine nachhaltige Nutzung der Wasserkraft. Österr Wasser- und Abfallw 65(9): 354-361-9. doi:10.1007/ s00506-013-0108-0

Hofer, B. (1987): Der Feststofftransport von Hochgebirgsbächen am Beispiel des Pitzbachs. Österr Wasser- und Abfallw 39(1): 29-38

Hofer, B. (1985): Der Feststofftransport von Hochgebirgsbächen am Beispiel des Pitzbachs. Dissertation, Universität Innsbruck.

Kammerlander, J. (2017 - unveröffentlicht): Long-term evolution of bed load transport in steep mountain streams and its link with the supply of mobile sediment. Eingereichte Dissertation, Universität Innsbruck.

Kammerlander, J., Gems, B., Kößler, D., Aufleger, M. (2017 - unveröffentlicht): Effect of bed load supply on sediment transport in mountain streams. Zur Veröffentlichung akzeptiert in Int J Sed Res.

Klar, R. (2016): Langzeitsimulation des Geschiebetransports in alpinen Tälern - Weiterentwicklung von Methoden zur Modellierung der langfristigen Sohllagenentwicklung und zur Ermittlung von Hochwassergefahren in inneralpinen Tälern. Dissertation, Universität Innsbruck.

Mao, L., Dell'Agnese, A., Huincache, C., Penna,

D., Engel, M., Niedrist, G., Comiti, F. (2014):
Bedload hysteresis in a glacier-fed mountain river. Earth Surf Proc Land 39: 964-976. doi:10. 1002/esp.3563

Nitsche, M., Rickenmann, D., Turowski, J. M. Badoux, A., Kirchner, J. W. (2011): Evaluation of bed load transport predictions using flow resistance equations to account for macro roughness in steep streams. Water Resour Res 47: W08513. doi:10.1029/2011WR010645

Parker, G., Klingeman, P. C. (1982): On why gravel bed streams are paved. Water Resour Res 18(5): 1409-1423.

Recking, A. (2012): Influence of sediment supply on mountain streams bedload transport Geomorphology 175-176: 139-150. doi:10.1016/ j.geomorph.2012.07.005

Rickenmann, D., Turowski, J. M., Fritschi, B. Wyss, C., Laronne, J., Barzilai, R., Reid, I., Kreisler, A., Aigner, J., Seitz, H., Habersack, H. (2014) Bed load transport measurements with impact plate geophones: comparison of sensor calibration in different gravel-bed streams. Earth Surf Proc Land 39(7): 928-942. doi:10.1002/esp.3499 Ryan, S. (1997): Morphologic response of subalpine sterams to transbasin flow diversion. J Am Water Resour Ass 33(4): 839-854.

Schneider, J. M., Rickenmann, D., Turowski, J. M., Kirchner, J. W. (2015a): Self-adjustmen of stream bed roughness and flow velocity in a steep mountain channel. Wat Resour Res 51: 7838-7859. doi:10.1002/2015WR016934

Schneider, J. M., Rickenmann, D., Turowski, J. M., Bunte, K., Kirchner, J. W. (2015b): Applicability of bed load transport models for mixedsize sediments in steep streams considering macro-roughness. Water Resour Res 51: 5260-5283. doi:10.1002/2014WR016417
Schober, W. (1961): Selbsttätige Entkiesungsund Entsandungsanlagen. Österreichische Wasserwirtschaft, Springer-Verlag, Wien, 99-108.

Schöberl, F. (1983): Statistische Untersuchun gen über die Geschiebeverfrachtung in Hochgebirgsbächen. Interpraevent 1983 (1): 74-85. Schönlaub, H., Hofer, B. (2009): Die Hochwassersituation bei abgeleiteten Bächen. Wasserwirtschaft 9: 23-29.

Sommer, N., Lauffer, H. (1982): Untersuchun gen über den Feststofftransport in Gebirgsbächen der Ostalpen, Proceedings of the 14th International Dam Conference, Rio de Janeiro, Brazil.

Sommer, N. (unveröffentlicht): Untersuchungen über die Kornverteilung (Sieblinien) des Geschiebes in Gebirgsbächen. Vorbericht der TIWAG: TB-7127/1.

Strobl, M. (1980): Schwebstoff- und Geschiebeführung in Gletscherbächen der zentralen Ötztaler Alpen (Tirol) und Untersuchung über den Gebirgsabtrag im Gebiet des Mittelbergferners. Dissertation, Universität Innsbruck.

Tschada, H. (1975): Beobachtungen über die Geschiebefracht von Hochgebirgsbächen. Interpraevent 1975 (1): 109-126.

Tschada, H., Hofer, B. (1990): Total solid load from the catchment area of the Kaunertal hydroelectric power station: the results of 25 years of operation. Hydrology in Mountain Regions II - Artificial Reservoirs, Water and Slopes, IAHS Publ. Nr. 194.

Yager, E. M., Turowski, J. M., Rickenmann, D. McArdell, B. W. (2012): Sediment supply, grain protrusion, and bed load transport in mountain streams. Geophys Res Lett 39: L10402. doi:10. 1029/2012GL051654 